\title{
Outcomes of novel surgical procedures for graft size mismatch in living-donor lobar lung transplantation
}

\author{
Daisuke Nakajima, MD, PhD, Satona Tanaka, MD, PhD, Akihiro Ohsumi, MD, PhD, and
}

Hiroshi Date, MD, PhD, Kyoto, Japan

From the Department of Thoracic Surgery, Kyoto University, Kyoto, Japan.

Disclosures: The authors reported no conflicts of interest.

The Journal policy requires editors and reviewers to disclose conflicts of interest and to decline handling or reviewing manuscripts for which they may have a conflict of interest. The editors and reviewers of this article have no conflicts of interest.

This study protocol was approved by the institutional review board of Kyoto University Hospital (R2389), and written informed consent was obtained from each participant.

Received for publication June 17, 2020; revisions received June 17, 2020; accepted for publication June 19, 2020; available ahead of print June 25, 2020.

Address for reprints: Hiroshi Date, MD, PhD, Department of Thoracic Surgery, Kyoto University, 54 Shogoinkawahara-cho, Sakyo-ku, Kyoto 606-8507, Japan (E-mail: hdate@kuhp.kyoto-u.ac.jp).

JTCVS Techniques 2020;3:388-91

2666-2507

Copyright (C) 2020 The Authors. Published by Elsevier Inc. on behalf of The American Association for Thoracic Surgery. This is an open access article under the CC BY-NC-ND license (http://creativecommons.org/licenses/bync-nd/4.0/).

https://doi.org/10.1016/j.xjtc.2020.06.037

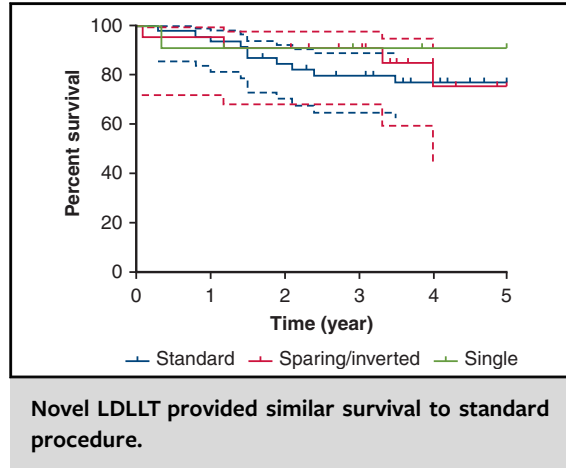

CENTRAL MESSAGE

Novel LDLLT such as native upper lobe-sparing, right-to-left inverted, and single-lobe transplants overcame graft size mismatch and showed good post-transplant pulmonary function and survival.

See Commentaries on pages $392,394,396$, and 398.
Various living-donor lobar lung transplantations (LDLLTs) have been developed to resolve the serious issue of graft size mismatch. ${ }^{1}$ The procedures of "native upper lobesparing transplant" and "right-to-left inverted transplant" have been employed as strategies for managing undersized grafts, and single-lobe transplant has been used to manage oversized grafts. ${ }^{1}$ The purpose of this study was to compare the outcomes of newly developed transplant techniques with those of the standard LDLLT approach.

\section{METHODS}

Between June 2008 and May 2018, we performed 47 standard LDLLT (standard group), 22 native upper lobe-sparing transplants and/or right-toleft inverted transplants for undersized grafts (sparing/inverted group), and 11 single-lobe transplants for oversized grafts (single group). Each transplant case was carefully reviewed and approved by the Lung Transplant Evaluation Committee at Kyoto University Hospital. The study protocol was approved by the institutional review board of Kyoto University Hospital (R2389), and written informed consent was obtained from each participant.

Each transplant procedure and inclusion criteria have been previously reported. ${ }^{1-3}$ In standard LDLLT, the right and left lower lobes were retrieved from 2 healthy donors and were implanted in a single recipient. In right-to-left inverted LDLLT, the graft bronchus was anastomosed to the recipient left upper lobe bronchus, leaving the recipient left lower bronchial stump closed. The recipient left bronchus was carefully dissected and the bronchial stump was reinforced with the pericardial fat pad to prevent the postoperative bronchopleural fistula. Pulmonary artery anastomosis was performed without twisting behind the bronchial anastomosis
(Figure 1). Native upper lobe-sparing $(\mathrm{n}=9)$ and right-to-left inverted transplants $(\mathrm{n}=11)$ were bilateral lung transplants in all cases except one. The native upper lobe-sparing transplant combined with right-toleft inverted transplant was performed in 2 cases. This surgical technique is presented in Video 1. Only right lower lobe was implanted in all single-lobe transplant cases. Among them, 4 patients required delayed chest closure and 1 patient simultaneously underwent left pneumonectomy.

For functional size matching, the graft forced vital capacity (FVC) was calculated based on the measured donor FVC and the number of resected pulmonary segments. An estimated graft FVC of $>45 \%$ to $50 \%$ of the recipient predicted $\mathrm{FVC}$ was usually considered to indicate acceptable size matching of the graft to the LDLLT recipient. ${ }^{4}$ For anatomical size matching, 3-dimensional computed tomography (CT) volumetry was performed.

The post-transplant outcomes and \% FVC and percent forced expiratory volume in 1 second of predicted values were compared among the 3 groups. All data are presented as mean \pm standard deviation. Survival was determined by a Kaplan-Meier survival analysis.

\section{RESULTS}

The patient characteristics are summarized in Table 1. Native upper lobe-sparing and/or right-to-left inverted 


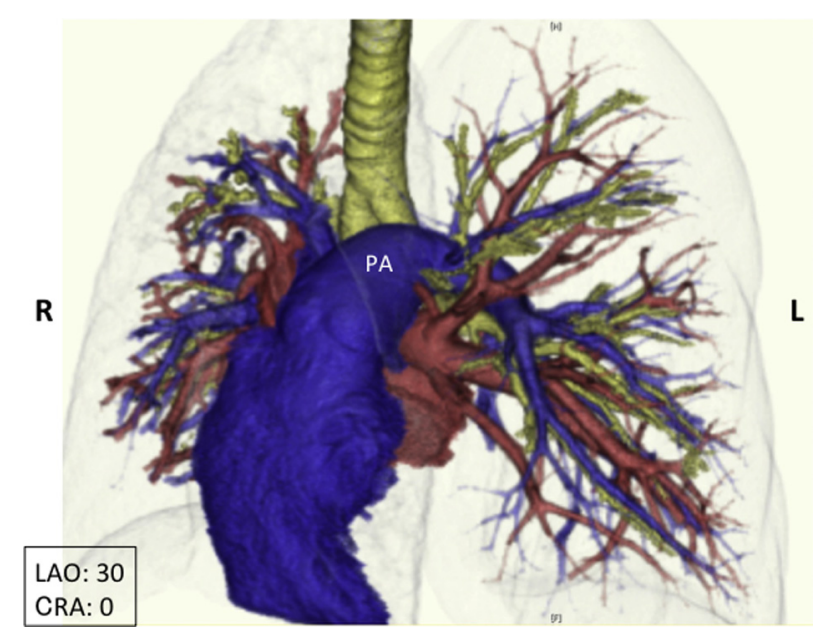

FIGURE 1. Three-dimensional computed tomography angiography showed no twisting and stenosis of the PA 1 month after ritht-to-left inverted transplantation. $P A$, Pulmonary artery; $L A O$, left anterior oblique view; $C R A$, cranial view.

transplants were performed mainly for large adult men, whereas most single-lobe transplants were performed in small pediatric patients. Although novel transplant procedures might be complicated in comparison to the standard LDLLT, the total graft ischemic time did not significantly differ among the $\mathrm{t} 3$ groups (standard: $118 \pm 26$ minutes; sparing/inverted: $126 \pm 25$ minutes; single: $116 \pm 32$ minutes).

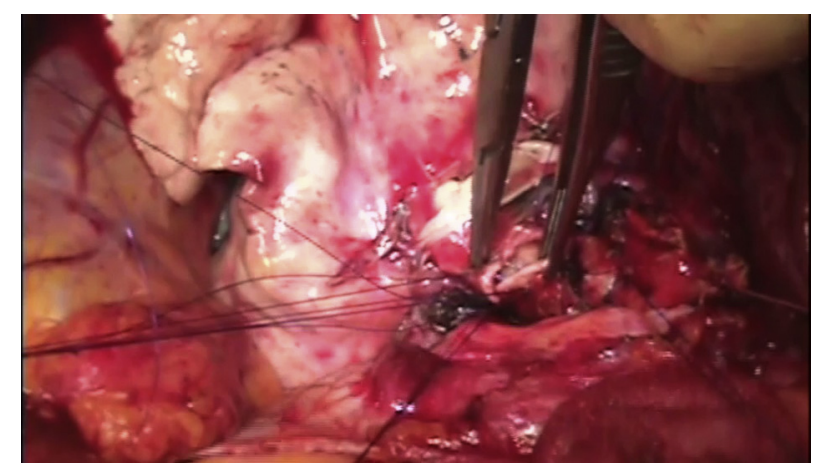

VIDEO 1. Native right upper lobe-sparing transplantation combined with right-to-left inverted transplantation for idiopathic pulmonary fibrosis. The first donor right lower lobe was inverted and implanted into the recipient left chest cavity. The graft bronchus was anastomosed to the recipient left upper lobe bronchus, pulmonary artery anastomosis was performed behind the bronchus, and the graft pulmonary vein was anastomosed to the recipient left upper pulmonary vein. Then, we spared the recipient right upper lobe and implanted the second donor right lower lobe into the recipient right thorax. The graft bronchus was anastomosed distally to the second carina, the graft pulmonary vein was anastomosed to the lower pulmonary vein, and the graft pulmonary artery was anastomosed to the interlobar artery. Video available at: https://www.jtcvs.org/article/S26662507(20)30319-9/fulltext.
TABLE 1. Recipient characteristics

\begin{tabular}{lccc}
\hline & $\begin{array}{c}\text { Standard } \\
(\mathbf{n}=\mathbf{4 7})\end{array}$ & $\begin{array}{c}\text { Sparing/inverted } \\
(\mathbf{n = 2 2})\end{array}$ & $\begin{array}{c}\text { Single } \\
(\mathbf{n}=\mathbf{1 1})\end{array}$ \\
\hline Age, y & $40 \pm 19$ & $41 \pm 14$ & $12 \pm 10$ \\
Children $<15$ y & $8(17 \%)$ & 0 & $10(91 \%)$ \\
Sex: male & $18(38 \%)$ & $15(58 \%)$ & $3(27 \%)$ \\
Height, cm & $155 \pm 13$ & $164 \pm 9$ & $118 \pm 12$ \\
Indications & & & \\
$\quad$ ILD & 22 & 10 & 1 \\
HSCT & 18 & 8 & 6 \\
PAH & 3 & 1 & 0 \\
CLAD & 2 & 1 & 1 \\
Others & 2 & $4(18.2 \%)$ & $3(27.3 \%)$ \\
Idiopathic or & $8(17.0 \%)$ & & \\
secondary & & & \\
PAH & & & \\
\hline
\end{tabular}

$I L D$, Interstitial lung disease; $H S C T$, pulmonary complication after hematopoietic stem cell transplantation; $P A H$, pulmonary arterial hypertension; $C L A D$, chronic lung allograft dysfunction.

Early post-transplant outcomes are shown in Table 2 . The single group showed significantly lower arterial oxygen tension/inspired oxygen fraction at intensive care unit admission than the standard or sparing/inverted group. Other early outcomes did not significantly differ among the 3 groups. Lung perfusion scintigraphy showed $86.7 \pm 2.4 \%$ perfusion to the transplanted right lower lobe at 1 year after transplantation in the single group.

In this study, the ratio of the graft volume to the recipient's chest cavity volume was $188 \pm 68 \%$ in the single group. Although the pretransplant FVC size matching between the donor graft and recipient was significantly lower in the sparing/inverted group $(51.8 \pm 9.6 \%$, Figure $2, A)$ than in the standard group $(69.1 \pm 17.9 \%)$ or single group $(66.8 \pm 18.2 \%)$, the pulmonary function at 1 year after transplantation was similar among the 3 groups: \%FVC of $59.4 \pm 22.1 \%$ in the standard group $(\mathrm{n}=40)$, $63.2 \pm 18.6 \%$ in the sparing/inverted group $(\mathrm{n}=19)$, and $60.5 \pm 21.2 \%$ in the single group $(\mathrm{n}=8)$ (Figure $2, B)$ and percent forced expiratory volume in 1 second of $58.7 \pm 19.9 \%$ in the standard group $(\mathrm{n}=41)$, $61.6 \pm 15.0 \%$ in the sparing/inverted $(\mathrm{n}=19)$, and $58.2 \pm 19.9 \%$ in the single group $(\mathrm{n}=7$ ) (Figure 2, $C$ ). The patients who underwent LDLLT had a favorable exercise capacity at 1 year after transplant, as demonstrated by the 6-minute walking distance (standard $[\mathrm{n}=39]$ : $474 \pm 132 \mathrm{~m}$; sparing/inverted [ $\mathrm{n}=20$ ]: $525 \pm 114 \mathrm{~m}$; single $[\mathrm{n}=6]: 399 \pm 81 \mathrm{~m}$, Figure 2, $D$ ).

Nobody was lost to follow-up. During the median followup period of 5.1 years, 10 patients died in the standard group, 4 patients died in the sparing/inverted group, and 1 patient died in the single group. The overall 5-year survival of LDLLT was $79.0 \%$, and each group showed an excellent 
TABLE 2. Early post-transplant outcomes

\begin{tabular}{|c|c|c|c|}
\hline & Standard $(n=47)$ & Sparing/inverted $(n=22)$ & Single $(\mathbf{n}=11)$ \\
\hline $\mathrm{PaO}_{2} / \mathrm{FIO}_{2}$ at ICU admission, $\mathrm{mm} \mathrm{Hg}$ & $475 \pm 130$ & $473 \pm 139$ & $316 \pm 141$ \\
\hline ECMO requirement & $5(10.6 \%)$ & 0 & $2(18.2 \%)$ \\
\hline Rethoracotomy indications & $\begin{array}{c}6(12.8 \%) \\
\text { hemothorax } 5 \text {, empyema } 1\end{array}$ & $\begin{array}{c}2(9.1 \%) \\
\text { hemothorax } 2\end{array}$ & $\begin{array}{r}2(18.2 \%) \\
\text { hemothorax } 2\end{array}$ \\
\hline Duration of mechanical ventilation, $d$ & $17 \pm 22$ & $13 \pm 10$ & $25 \pm 20$ \\
\hline Tracheostomy & $27(57.4 \%)$ & $16(72.7 \%)$ & $6(54.5 \%)$ \\
\hline Bronchial complication & $4(8.5 \%)$ & $1(4.5 \%)$ & 0 \\
\hline 30-d mortality & $1(2.1 \%)^{*}$ & $1(4.5 \%) \dagger$ & 0 \\
\hline In-hospital mortality & $2(4.3 \%) \ddagger$ & $1(4.5 \%)$ & $1(9.1 \%) \dagger$ \\
\hline
\end{tabular}

Cause of death: *thrombosis; †primary graft dysfunction; łaspiration pneumonitis. $\mathrm{PaO}_{2} / \mathrm{FIO}_{2}$, Arterial oxygen tension/inspired oxygen fraction; $\mathrm{ICU}$, intensive care unit; $\mathrm{ECMO}$, extracorporeal membrane oxygenation.

5-year survival (standard: $77.0 \%$; sparing/inverted: 75.4\%; single: $90.9 \%$, Figure 3).

The donor postoperative outcome is one of the most important things in LDLLT. We performed lobectomy in

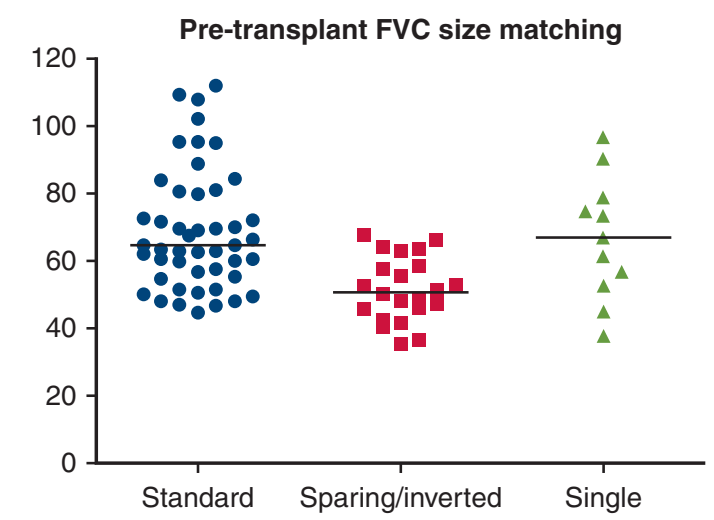

A

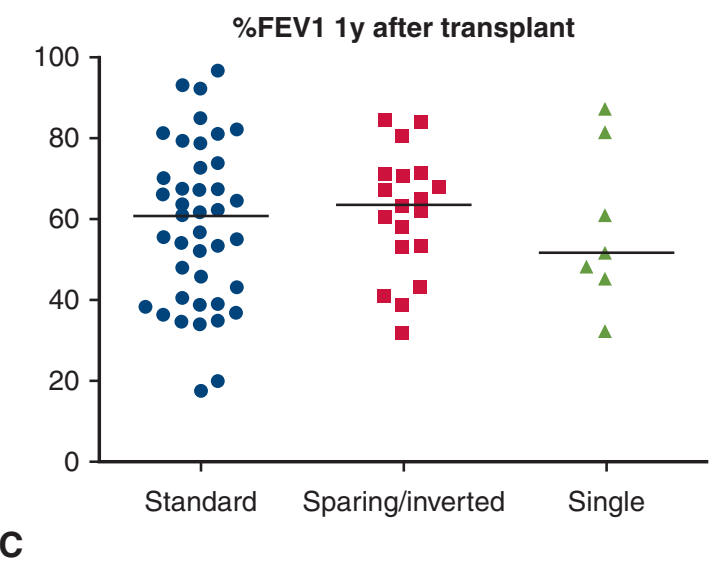

148 live donors in this study. Postoperative complications were observed in 22 donors $(14.9 \%)$, including pneumothorax $(\mathrm{n}=8)$, pleural effusion $(\mathrm{n}=7)$, pleuritis $(\mathrm{n}=3)$, chylothorax $(\mathrm{n}=2)$, hemothorax $(\mathrm{n}=1)$, and empyema

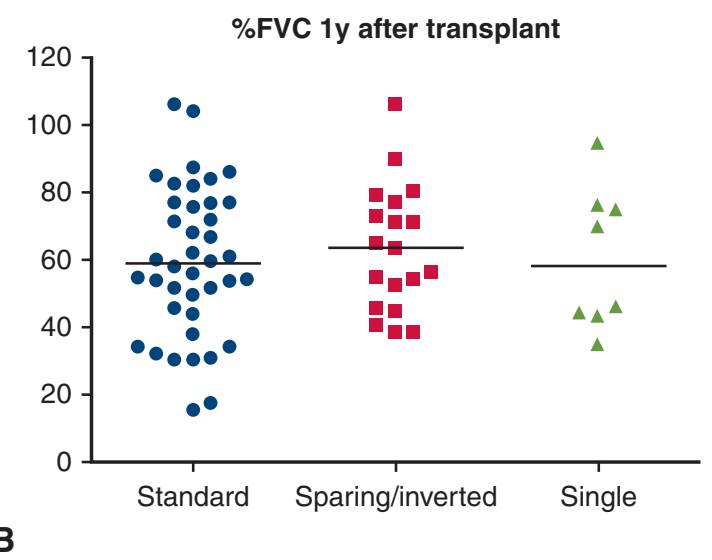

6 minute walking distance 1 y after transplant $(\mathrm{m})$

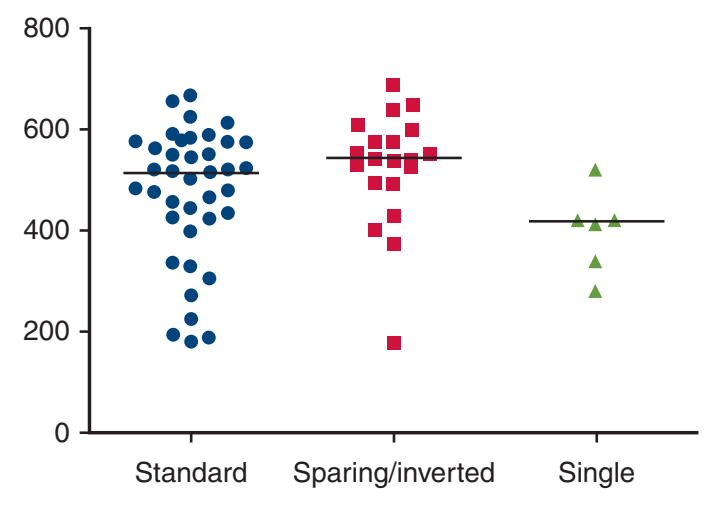

FIGURE 2. We performed 47 standard living-donor lobar lung transplants (standard), 22 native upper lobe-sparing transplants and/or right-to-left inverted transplants for undersized grafts (sparing/inverted), and 11 single-lobe transplants for oversized grafts (single). A, The FVC size matching between the graft and recipient was significantly lower in the sparing/inverted group $(51.8 \pm 9.6 \%)$ before transplantation than in the standard group $(69.1 \pm 17.9 \%)$ or single group $(66.8 \pm 18.2 \%)$. The percent FVC of predicted value (B), \% FEV1 (C), and 6-minute walking distance (D) were similar among the 3 groups at 1 year after transplantation. FVC, Forced vital capacity; \% FEV1, \% forced expiratory volume in 1 second. 


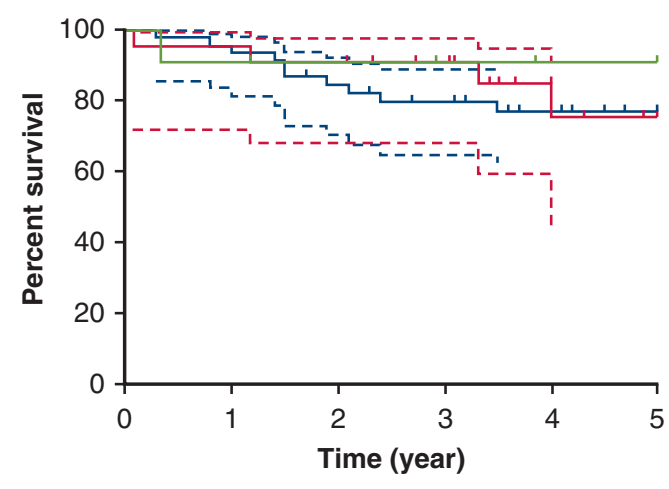

+ Standard + Sparing/inverted + Single

\begin{tabular}{|l|c|c|c|c|c|c|}
\hline $\begin{array}{l}\text { \# of patients } \\
\text { at risk }\end{array}$ & 0 & $1 y$ & $2 y$ & $3 y$ & $4 y$ & $5 y$ \\
\hline Standard & 47 & 44 & 37 & 33 & 27 & 22 \\
\hline Sparing/inverted & 22 & 22 & 21 & 18 & 9 & 5 \\
\hline Single & 11 & 11 & 10 & 9 & 8 & 7 \\
\hline
\end{tabular}

FIGURE 3. The overall 5-year survival was $77.0 \%$ after standard living-donor lobar lung transplantation (standard), $75.4 \%$ after native upper lobe-sparing transplantation and/or right-to-left inverted transplantation (sparing/inverted), and $90.9 \%$ after single-lobe transplantation (single). The dashed lines are the lower and upper $95 \%$ confidence interval limits.

$(\mathrm{n}=1)$. Importantly, all donors survived and returned to their previous lifestyle.

\section{DISCUSSION}

Native upper lobe-sparing and/or right-to-left inverted techniques have been employed for undersized grafts. In native upper lobe-sparing transplantation, the spared upper lobes can reduce intrathoracic dead space after implantation and provide an adequate chest cavity for the graft so that the undersized lobar graft can be ventilated more efficiently. ${ }^{3}$ In right-to-left inverted transplantation, we can implant the $25 \%$ larger right lower lobe instead of the left lower lobe into the recipient's left chest cavity. ${ }^{2}$ In the present study, the preoperative FVC size matching was significantly increased from $54.0 \pm 10.2 \%$ in a noninverted setting to $59.7 \pm 10.3 \%$ in an inverted setting. Therefore, the sparing/inverted group showed an equivalent pulmonary function to the standard group after transplantation despite the significant size discrepancy between the undersized graft and recipient.

Functional and anatomical size matching between oversized grafts and small patients is important in single-lobe transplantation. We previously reported that patients who received grafts with an FVC of $<60 \%$ and a CT volume of $>170 \%$ developed severe primary graft dysfunction after single-lobe transplantation. ${ }^{5}$ Furthermore, when the graft volume is $<200 \%$ of the recipient chest cavity volume, the graft can be fitted into the small chest cavity of the recipient. In the present study, the mean FVC size matching was $66.8 \%$ and the mean $\mathrm{CT}$ volumetry size matching was $188 \%$ in the single group, which might result in better early post-transplant outcomes and survival than those previously reported values. ${ }^{5}$

\section{CONCLUSIONS}

Despite its small sample size, this preliminary study includes the broadest experience of LDLLT to describe the utility of novel transplant procedures to deal with graft size mismatch.

\section{References}

1. Date H, Aoyama A, Hijiya K, Motoyama H, Handa T, Kinoshita H, et al. Out comes of various transplant procedures (single, sparing, inverted) in living donor lobar lung transplantation. J Thorac Cardiovasc Surg. 2017;153:479-86.

2. Chen F, Miyamoto E, Takemoto M, Minakata K, Yamada T, Sato M, et al. Righ and left inverted lobar lung transplantation. Am J Transplant. 2015;15:1716-21.

3. Takahagi A, Chen-Yoshikawa TF, Saito M, Okabe R, Gochi F, Yamagishi H, et al Native upper lobe-sparing living-donor lobar lung transplantation maximizes respiratory function of the donor graft. J Hear Lung Transplant. 2019;38:66-72.

4. Date H, Aoe M, Nagahiro I, Sano Y, Andou A, Matsubara H, et al. Living-donor lobar lung transplantation for various lung diseases. J Thorac Cardiovasc Surg. 2003;126:476-81.

5. Date H, Shiraishi T, Sugimoto S, Shoji T, Chen F, Hiratsuka M, et al. Outcome of living-donor lobar lung transplantation using a single donor. J Thorac Cardiovasc Surg. 2012;144:710-5 